\title{
Coordenação global, equilíbrio, índice de massa corporal e nível de atividade física: um estudo correlacional em idosos de lvoti, RS, Brasil
}

\author{
Gobal cordnation posturd balance, booymassindex andleved of physcd ativity. a \\ correational study in edalys abjetsfromlvat, RS Brazl
}

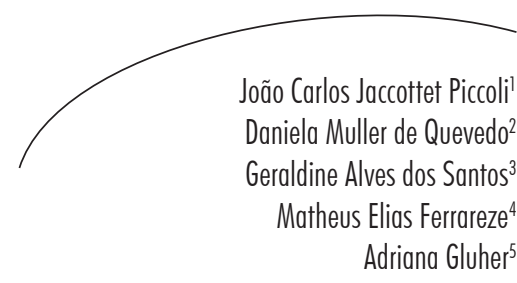

Resumo

Objetivo: Buscou-se verificar se existe correlação entre o índice de massa corporal, nível de atividade física em METs, coordenação global e equilíbrio estático de idosos da cidade de Ivoti, RS. Materiais e Métodos: estudo descritivo de corte transversal que avaliou 202 idosos, de ambos os gêneros, residentes em Ivoti-RS e selecionados por conveniência. Para as avaliações foram utilizados testes de coordenação global e de equilíbrio, o Questionário Internacional de Atividade Física (IPAQ) formato curto, e calculado o Índice de Massa Corporal segundo os pontos de corte propostos: desnutrição: $<22,0 \mathrm{~kg} / \mathrm{m}^{2}$, eutrofia: de 22,0 a $27,0 \mathrm{~kg} / \mathrm{m}^{2}$ e obesidade: $>27,0 \mathrm{~kg} / \mathrm{m}^{2}$. O nível de atividade foi classificado em Mets-min/semana como: muito ativo de 1500 a 3000 Mets-min./semana; suficientemente ativo, entre 1499 e 600 Mets-min./ semana e insuficientemente ativo, menos de 600 Mets-min./semana. Os dados foram submetidos à estatística descritiva e correlação de Spearman $(\alpha=0,05)$, através do programa SPSS, versão 16.0. O estudo foi aprovado pelo CEP da Universidade Feevale, Novo Hamburgo, RS. Resultados: A amostra foi composta por $62(30,7 \%)$ homens e $140(69,3 \%)$ mulheres, idade média masculina de 69,53 \pm 6,23 e feminina de 67,52 \pm 5,69 anos. A idade média geral da amostra foi de 68,14 \pm 5,92 anos. Foram verificadas associações negativas entre as variáveis IMC e coordenação global e IMC e equilíbrio, $\mathrm{r}=$ $-0,423(p<0,01)$ e $\mathrm{r}=-0,3060(p<0,01)$ respectivamente. Porém, quando estratificadas por gênero, essas associações não foram significativas nos homens. O nível de atividade física demonstrou correlação negativa com a variável IMC $(\mathrm{r}=-0,219)$ e com a idade ( $\mathrm{r}=$ -0,241); da mesma forma, apresentou correlação positiva com a coordenação motora

\footnotetext{
Programa de Pós-Graduação em Inclusão Social e Acessibilidade / Curso de Educação Física. Universidade Feevale. Novo Hamburgo, RS, Brasil.

2 Programa de Pós-Graduação em Qualidade Ambiental. Universidade Feevale. Novo Hamburgo, RS, Brasil.

Programa de Pós-Graduação em Inclusão Social e Acessibilidade / Curso de Psicologia. Universidade Feevale. Novo Hamburgo, RS, Brasil.

4 Curso de Educação Física, Instituto de Ciências da Saúde. Universidade Feevale. Novo Hamburgo, RS, Brasil.

5 Curso de Nutrição, Instituto de Ciências da Saúde. Universidade Feevale. Novo Hamburgo, RS, Brasil.
}

Palavras-chave: Idoso. Índice de Massa Corporal. Equilíbrio postural. Nível de atividade física. Atividade Motora, Ivoti, RS. 
( $r=0,274)$ e o equilíbrio ( $r=0,191)$. Mas o IMC e o equilíbrio, assim como a idade e o equilíbrio, não apresentaram correlação nas pessoas idosas suficientemente ativas. A coordenação global e o equilíbrio estão relacionados positivamente ao nível de atividade física apenas nas mulheres. Entretanto, o IMC está relacionado negativamente ao nível de atividade física em ambos os sexos. Conclusão: Para as mulheres da amostra, o alto valor do IMC gerou possivelmente resultados inferiores no desempenho dos testes de coordenação global e equilíbrio. Tal fato não pôde ser observado entre os homens, mas constatou-se correlação positiva entre o nível de atividade física em METs totais, coordenação global e equilíbrio na amostra total do estudo.

\section{Abstract}

Objective: This study aimed to assess the relationship among Body Mass Index (BMI), physical activity level, global coordination, and postural balance in an elderly population from Ivoti, state of Rio Grande do Sul, Brazil. Methods: This descriptive cross-sectional study assessed a convenience sample of 202 elderly female and male subjects. It was used the global coordination test and the postural balance test and calculated the BMI according to the cutoff points: malnutrition: $<22.0 \mathrm{~kg} / \mathrm{m}^{2}$, normal weight: from 22.0 to $27.0 \mathrm{~kg} / \mathrm{m}^{2}$, and obesity: $>27.0 \mathrm{~kg} / \mathrm{m}^{2}$. The level of physical activity was classified in METs as: high active from 1,500 to 3,000 Mets-min/week; moderate from 600 to $1499 \mathrm{Mets}-\mathrm{min} /$ week and low below $600 \mathrm{Mets}-\mathrm{min} /$ week. Data was submitted to the descriptive statistical and the Spearman correlation $(\alpha=0.05)$ using the SPSS software, version 16.0. The study was approved by the Ethics Research Committee of Universidade Feevale, Novo Hamburgo, RS, Brazil. Results: Among the 202 individuals, $62(30.7 \%)$ were males and 140 (69.3\%), females. The male and female age averages were $69.53 \pm 6.23$ and $67.52 \pm 5.69$ years old, respectively. The general sample age average was $68.14 \pm 5.92$ years. Negative correlation between BMI and global coordination and BMI and balance was detected, $r=-0.423(\mathrm{p}<0.01)$ and $\mathrm{r}=-0.3060(\mathrm{p}<0.01)$, respectively. However, when stratified by sex, these associations were not significant for males. The physical activity level showed negative correlation when compared with BMI $(r=-0,219)$ and with age $(r=-0,241)$, it had a positive correlation when compared with global coordination $(r=0,274)$ and balance $(r=0,191)$. When compared BMI and balance and age and balance no correlation was found in the elderly subjects classified as sufficiently active. Global coordination and balance were positively correlated to the level of physical activity only in female subjects. However, BMI is negatively correlated to the level of physical activity of both genders. Conclusion: it was concluded that the sample women's high BMI may have produced worse performance on the global coordination and balance tests. There was significant moderate negative correlation between level of physical activity in total METs and BMI of both men and women and BMI, global coordination, and postural balance only among the female elderly sample. However, a positive correlation among level of physical activity in total METs, global coordination, and postural balance of the studied sample was observed.
Key words: Aged. Body Mass Index. Postural balance. Level of physical activity. Motor Activity. Ivoti city. 


\section{INTRODUÇÃO}

O envelhecimento é um conjunto de processos que ocorre nos seres vivos, resultando no decréscimo das capacidades motoras, redução da força, flexibilidade e velocidade, além da redução dos níveis de $\mathrm{VO}_{2}$ máximo ${ }^{1,2}$. Spirduso ${ }^{3}$ afirma, também existir, neste ciclo de vida, a perda de adaptabilidade e deficiência funcional, e Rosa $\mathrm{Neto}^{4}$ relata que o risco de incapacidade, a deterioração da qualidade de vida e a perda da independência são processos que dele fazem parte.

Além do aumento do número de idosos, é possível identificar um crescente aumento no índice de sobrepeso e obesidade na população mundial. Segundo a Organização Mundial da Saúde (OMS), ${ }^{5}$ há cerca de um bilhão de adultos que apresentam sobrepeso corporal, e pelo menos 300 mil são obesos. Geralmente associados à má alimentação e baixo nível de atividade física, o sobrepeso e a obesidade contribuem de forma importante para o aumento das doenças crônicas e diminuição das capacidades físicas do indivíduo. ${ }^{6}$

Com o avanço da idade, é visível a mudança nas dimensões corporais, tanto no que se refere à estatura como nas variáveis relacionadas à composição corporal. ${ }^{1}$ Em homens, as alterações no índice de massa corporal (IMC) atingem seu valor máximo entre 45 e 49 anos, apresentando em seguida um ligeiro declínio. Já nas mulheres, esse pico se dá entre 60 e 70 anos. $^{3}$ A associação do aumento do IMC com inúmeros riscos à saúde é contínua, mas sua interpretação difere de acordo com as populações analisadas, uma vez que os valores de referência mudam de acordo com os grupos etários e o gênero em questão.

Mazo et al. ${ }^{7}$ investigaram a aptidão funcional geral e o índice de massa corporal em um grupo de 100 idosas, entre 60 e 79 anos, participantes do Grupo de Estudos da Terceira Idade (GETI), da Universidade de Santa Catarina (UDESC). No estudo, o critério de inclusão dos sujeitos era que estivessem participando pelo menos há seis meses nas atividades de hidroginástica $(\mathrm{n}=75)$ e natação $(\mathrm{n}=25)$. Os participantes foram submetidos à bateria de testes para idosos da American Alliance for Health, Physical Education, Recreation and Dance (AAHPERD), que avaliou a coordenação, a resistência de força, a flexibilidade, a agilidade, o equilíbrio dinâmico e a resistência aeróbia em relação ao índice de massa corporal. Foi então identificada uma tendência de 42,9\% das idosas com um Índice de Aptidão Funcional Geral (IAFG) classificado na categoria "bom", terem um IMC normal e 95\% das que apresentavam um IAFG "fraco", de apresentarem um IMC de sobrepeso. Observouse uma relação inversa entre o IMC e a aptidão funcional geral em idosas, isto é, níveis mais altos de IMC estavam relacionados a performances mais baixas em testes de aptidão funcional. Assim, a manutenção de níveis normais de IMC e bons, em testes de aptidão funcional, pode ser importante não apenas para a prevenção de doenças, mas também para a manutenção da autonomia e independência do idoso.

Outro estudo foi desenvolvido com uma subamostra de 501 idosos de 60 a 93 anos, entrevistados no Projeto Processos do Envelhecimento Saudável (PENSA) de um total de 956 residentes da cidade de Juiz de Fora, MG. A subamostra correspondia a indivíduos que responderam a perguntas qualitativas sobre a definição de envelhecimento saudável e meios de alcançar esse envelhecimento. Constatouse, na visão dos idosos investigados, que um envelhecimento saudável estava relacionado à: a) manutenção da saúde física (53\%); b) saúde social (46\%); c) saúde emocional (37\%); d) preocupação com alimentação/exercícios (36\%); e d) evitar fatores de risco (19\%). Com esse estudo, pôdese identificar a relação feita de envelhecimento saudável com a ausência de perdas físicas, sociais e emocionais. ${ }^{8}$

O processo de envelhecimento pode provocar, então, modificações que levam ao declínio da capacidade funcional e ao aparecimento de uma ou mais doenças crônicas. É preciso compreender que a velhice não é sinônimo de doença e que o envelhecimento ativo ou a velhice bem-sucedida representam um potencial a ser desenvolvido no ser humano. Portanto, os déficits precisam ser 
compreendidos e prevenidos, ou em último caso tratados adequadamente, pois eles irão interferir de maneira significativa na qualidade de vida do idoso. Um elemento importante na discussão do envelhecimento ativo é o sedentarismo e o risco de quedas.

De acordo com a OMS, ${ }^{9}$ o consumo excessivo de calorias eleva significativamente o risco do aparecimento de obesidade, doenças crônicas e deficiências no decorrer do processo de envelhecimento. $\mathrm{O}$ material desenvolvido pela OMS sobre o envelhecimento ativo aponta:

dietas ricas em gordura (saturada) e sal, pobres em frutas e legumes/verduras e que suprem uma quantidade insuficiente de fibras e vitaminas, combinadas ao sedentarismo, são os maiores fatores de risco de problemas crônicos, como diabete, doença cardiovascular, pressão alta, obesidade, artrite e alguns tipos de câncer ${ }^{9}$ (p. 24).

A partir da contextualização feita anteriormente, pode-se verificar a necessidade de o idoso manter níveis normais de IMC, praticar atividade física regularmente e apresentar bons níveis de coordenação e equilíbrio, não apenas para a prevenção de doenças, mas para a manutenção de sua autonomia e independência. Assim, o presente estudo teve por objetivo verificar se existe correlação entre o índice de massa corporal, nível de atividade física em METs, coordenação global e equilíbrio estático de idosos da cidade de Ivoti, RS.

\section{MATERIAIS E MÉTODOS}

Este estudo, de característica descritiva e de corte transversal, utilizou uma amostra de 202 indivíduos de ambos os sexos, aparentemente saudáveis, com idades entre 60 e 83 anos, moradores da cidade de Ivoti, RS, selecionados por conveniência a partir de uma amostragem não probabilística. Os participantes foram distribuídos em duas classes: classe I, de 60 a 69 anos e classe II, a partir de 70 anos. A amostra foi então composta por 140 idosos do sexo feminino e 62 do masculino.
Inicialmente, os participantes foram contatados pessoalmente ou por telefone para o agendamento da coleta de dados, e comunicados a respeito do transporte que seria oferecido até o local das coletas, o Campus II da Universidade Feevale, Novo Hamburgo, RS. Todos os que se propuseram a participar do estudo foram devidamente esclarecidos sobre os propósitos da investigação e assinaram voluntariamente um termo de consentimento livre e esclarecido. O estudo foi aprovado pelo Comitê de Ética em Pesquisa da Universidade Feevale, Novo Hamburgo, RS, sob número 4.08.01.06.465.

Como instrumento de estudo, foi utilizada a Escala Motora para a Terceira Idade (EMTI), ${ }^{4}$ que define a aptidão motora geral a partir das seis subdivisões que se seguem: Motricidade Fina (capacidade de realizar movimentos de preensão e controle motor de precisão), Coordenação Global (movimentos de coordenação motora ampla), Equilíbrio (movimentos de estabilidade corporal e propriocepção), Esquema Corporal e Rapidez (representação corporal), Organização Espacial (percepção de direita e esquerda) e Organização Temporal (percepção do tempo, ritmo, velocidade e memória). A fim de responder aos objetivos propostos pelo estudo, foram selecionadas para análise apenas duas das variáveis aqui citadas, coordenação global e equilíbrio estático, por se pressupor que estas apresentassem associação direta com as variáveis IMC e nível de atividade física em METs.

A avaliação das provas de coordenação global e de equilíbrio estático foi composta por testes distribuídos em dez níveis de dificuldades cada um. À medida que o idoso tivesse sucesso num determinado nível da prova de coordenação global, recebia um resultado positivo registrado numa planilha e progredia para o nível seguinte. No momento em que não lograsse sucesso no nível de dificuldade subsequente, o idoso não avançava mais na prova em questão, passando para a seguinte, a de equilíbrio estático, seguindo o mesmo procedimento.

A prova de coordenação global era composta pelos seguintes testes, distribuídos em níveis de 
dificuldades: a) subir num banco; b) saltar sobre uma corda; c) saltar no mesmo lugar; d) saltar uma altura de $20 \mathrm{~cm}$; e) caminhar em linha reta; f) fazer o "pé manco"; g) saltar uma altura de 40 cm; h) saltar no ar; i) fazer o "pé manco" com uma caixa de fósforos; e j) saltar sobre uma cadeira. ${ }^{4}$

$\mathrm{Na}$ avaliação do equilíbrio estático, foram utilizados os seguintes testes: a) equilíbrio sobre um banco; b) equilíbrio sobre um joelho; c) equilíbrio com o tronco flexionado; d) equilíbrio nas pontas dos pés; e) equilíbrio através do "pé manco" estático; f) equilíbrio através da execução do quadro; g) equilíbrio de cócoras; h) equilíbrio com o tronco flexionado sobre as pontas dos pés; i) equilíbrio nas pontas dos pés com olhos fechados; e j) equilíbrio através do "pé manco" estático de olhos fechados. ${ }^{4}$

As provas motoras de coordenação global e de equilíbrio estático geraram uma pontuação por teste que variava de 12 a 132 pontos, de acordo com os níveis motores de dificuldade em que se classificavam os idosos. A classificação das provas de coordenação global e de equilíbrio atendeu à seguinte pontuação: $\geq 130$ (muito superior); 120 a 129 (superior); 110 a 119 (normal alto); 90 a 109 (normal médio); 80 a 89 (normal baixo); 70 a 79 (inferior); e $\leq 69$ (muito inferior). Para se determinar o nível de atividade física dos sujeitos da amostra na última semana, utilizouse a versão 8 do Questionário Internacional de Atividade Física (IPAQ), versão curta, ${ }^{10}$ que foi administrado através de entrevistas. Este é composto por questões sobre as atividades físicas moderadas, vigorosas e caminhadas praticadas pelos sujeitos do estudo nos sete dias anteriores à aplicação do instrumento. Para analisar os dados relativos ao nível de atividade física, foram utilizados os critérios de frequência e duração, classificando os indivíduos em três categorias: muito ativo, suficientemente ativo e insuficientemente ativo. ${ }^{11}$ Trata-se de um instrumento desenvolvido sob a chancela da OMS, ${ }^{11}$ com a finalidade de estimar o nível de prática habitual de atividade física através de oito questões. Suas informações permitem estimar o tempo despendido por semana em diferentes dimensões de atividade física (caminhadas e esforços físicos de intensidades moderada e vigorosa) e de inatividade física (posição sentada), com os resultados transformados em METs (equivalente metabólico), quantidade de energia gasta por um corpo enquanto em repouso.

A partir desse estado, os METs são aumentados à medida que a intensidade da atividade for também, aumentada. A unidade de medida MET-min por semana equivale ao nível de MET relacionado a atividade (caminhada, atividades moderadas e vigorosas) multiplicado pelo tempo gasto em minutos multiplicado pela frequência semanal de prática da atividade física. Assim, a caminhada equivale a 3,3 METs multiplicado por 30 min multiplicado por cinco dias por semana = 495 METs-min/sem.; as atividades moderadas equivalem a 4,0 METs multiplicado por 30 min multiplicado por cinco dias por semana $=600 \mathrm{METs}-\mathrm{min} / \mathrm{sem} ;$ as atividades vigorosas equivalem a 8,0 METs multiplicado por 30min multiplicado por cinco dias por semana $=1.200$ METs-min/sem. Para a classificação dos sujeitos nos níveis de atividade física, utilizaram-se as categorias oficiais do IPAQ: insuficientemente ativos - aqueles sujeitos que não se enquadrassem em nenhuma das categorias que se seguem ou que não praticassem atividades físicas; suficientemente ativos - aqueles sujeitos que praticassem três ou mais dias de atividade vigorosa de no mínimo 20 minutos por dia ou cinco ou mais dias de atividades moderadas ou caminhadas de no mínimo 30 minutos por dia ou cinco ou mais dias de qualquer combinação de caminhada, atividades moderadas ou vigorosas, alcançando o mínimo de $600 \mathrm{MET}-\mathrm{min} / \mathrm{sem}$ e muito ativos aqueles sujeitos que praticassem no mínimo três dias de atividades vigorosas e acumulassem um mínimo de 1.500 MET-min/semana ou sete ou mais dias de qualquer combinação de caminhada, atividade moderada ou vigorosa alcançando no mínimo 3.000 METs-min/semana.

Para calcular o índice de massa corporal (IMC), foi utilizada uma balança de equilíbrio de marca Wellmy com precisão de $100 \mathrm{~g}$. Durante a verificação, solicitou-se aos sujeitos que utilizassem roupas leves e estivessem descalços. A variável estatura foi medida utilizando-se um 
estadiômetro de mesma marca com precisão de $0,1 \mathrm{~cm}$, estando os indivíduos descalços e com a cabeça posicionada no plano de Frankfurt. Calculou-se o IMC considerando-se a razão massa corporal total $(\mathrm{kg})$ e o quadrado da estatura (m) e os pontos de corte utilizados foram os propostos por Lipschitz: ${ }^{12}$ desnutrição: $<22,0 \mathrm{~kg} / \mathrm{m}^{2}$, eutrofia: de 22,0 a $27,0 \mathrm{~kg} / \mathrm{m}^{2}$ e obesidade: $>27,0 \mathrm{~kg} / \mathrm{m}^{2}$.

Os testes utilizados neste estudo foram administrados em diferentes salas na Universidade Feevale, Novo Hamburgo, RS, por um avaliador que os aplicava individualmente e auxiliava os idosos quando necessário, e outro que registrava os resultados. O tempo médio total de aplicação de todos os testes foi de 45 minutos. Para a análise dos dados, foram utilizados a análise descritiva através de média, desvio padrão e coeficiente de variação para todas as variáveis do estudo; e para avaliar a correlação entre o IMC, coordenação global e o equilíbrio, utilizou-se o teste de correlação não-paramétrico de Spearman. Optou-se pelo teste não-paramétrico devido à não-normalidade dos dados, sendo para isto utilizado o teste de Kolmogorov-Smirnov (tabela 1). Os testes foram realizados a partir do pacote estatístico SPSS, versão 16.0, considerando-se o nível de significância de 5\%.

\section{RESULTADOS}

O estudo apontou que dos 202 sujeitos participantes, $69,3 \% \quad(\mathrm{n}=140)$ eram mulheres e 30,7\% ( $n=62)$ homens, estando entre 60 e 83 anos de idade, todos residentes na cidade de Ivoti, RS. A idade média apresentada pelos 202 indivíduos foi de 68,14 \pm 5,92 anos, sendo que os homens apresentaram idade média de 69,53 \pm 6,23 e as mulheres, 67,52 \pm 5,69 anos.

Tabela 1 - Distribuição da média, desvio-padrão (DP), coeficientes de variação (CV), valores mínimos e máximos referentes ao IMC, MET total e às variáveis Coordenação Global e Equilíbrio dos sujeitos da amostra na Escala Motora para a Terceira Idade e teste de normalidade de Kolmogorov-Smirnov $(n=202)$. Ivoti, RS.

\begin{tabular}{lccccccc}
\hline \multicolumn{1}{c}{ Variáveis } & Média & DP & CV & Mínimo & Máximo & KS & p-value \\
\hline IMC & 28,90 & 4,89 & 16,92 & 18,70 & 45,60 & 0,072 & $<0,012$ \\
METS totais & 2349 & 27,24 & 1,16 & 0 & 16158 & 0,186 & $<0,010$ \\
Coordenação Global & 58,60 & 30,56 & 52,15 & 12,00 & 120,00 & 0,096 & $<0,010$ \\
Equilíbrio & 73,75 & 35,14 & 47,65 & 12,00 & 132,00 & 0,111 & $<0,010$ \\
\hline
\end{tabular}

De acordo com os dados apresentados na tabela 1, no teste de coordenação global os participantes do estudo apresentaram média inferiore maior variação $(\mathrm{CV})$, quando comparada à variável equilíbrio, sendo classificados como "muito inferior" e "inferior", respectivamente, de acordo com os pontos de corte propostos por Rosa Neto. ${ }^{4}$

Considerando-se a variável IMC, constatouse que o índice médio foi equivalente a 28,9 $\mathrm{kg} / \mathrm{m}^{2}$ (tabela 1). Quando os sujeitos foram separados por gênero, os homens apresentaram índice médio de $27,0 \pm 3,8 \mathrm{~kg} / \mathrm{m}^{2}$ e as mulheres de $30,2 \pm 7,4 \mathrm{~kg} / \mathrm{m}^{2}$. Partindo-se dos pontos de corte utilizados como referência, isto é, aqueles propostos por Lipschitz ${ }^{12}$ - desnutrição: $<22,0$ $\mathrm{kg} / \mathrm{m}^{2}$, eutrofia: de 22,0 a $27,0 \mathrm{~kg} / \mathrm{m}^{2}$ e obesidade: $>27,0 \mathrm{~kg} / \mathrm{m}^{2}-$, a amostra do presente estudo classificou-se como obesa.

Foi testada a normalidade dos dados através do teste de Kolmogorov-Smirnov (KS), que apresentou significância inferior a 0,05 para as variáveis do estudo, constatando-se, assim, sua anormalidade. 
A tabela 2 apresenta os níveis de correlação entre as variáveis IMC e coordenação global e IMC e equilíbrio, considerando a divisão entre os sexos e os indivíduos como um todo. A partir desta tabela, é possível observar que houve correlação negativa moderada entre as variáveis IMC e coordenação global, assim como entre IMC e equilíbrio $(p \leq 0,01)$, quando considerada a amostra total do estudo. Quando analisado apenas o grupo masculino, não foi encontrado qualquer tipo de correlação estatisticamente significativa entre as variáveis, mas no grupo das mulheres participantes, maioria da amostra, é possível verificar uma correlação negativa moderada significativa entre as variáveis analisadas $(p \leq 0,01)$.

Tabela 2 - Nível de correlação e significância entre as variáveis IMC e AM2 e IMC e AM3 dos sujeitos divididos por sexo ( $\mathrm{n}=202)$. Ivoti, RS.

\begin{tabular}{lcccc}
\hline & \multicolumn{2}{c}{ Coordenação global } & \multicolumn{2}{c}{ Equilíbrio } \\
\cline { 2 - 5 } IMC & Rho $(\varrho)$ & $P$ & Rho $(\varrho)$ & $P$ \\
\hline Homens $(\mathrm{n}=62)$ & $-0,229$ & 0,073 & $-0,147$ & 0,254 \\
Mulheres $(\mathrm{n}=140)$ & $-0,453^{* *}$ & 0,000 & $-0,370^{* *}$ & 0,000 \\
Total $(\mathrm{n}=202)$ & $-0,423^{* *}$ & 0,000 & $-0,306^{* *}$ & 0,000 \\
\hline
\end{tabular}

${ }^{* *} p \leq 0,01$

A tabela 3 apresenta os níveis de correlação entre a variável MET total, IMC, coordenação global e equilíbrio estático dos indivíduos como um todo. Estes permitem observar que houve correlação negativa moderada entre as variáveis MET total e IMC $(p \leq 0,01)$. A variável MET total e coordenação global e MET total e equilíbrio apresentaram correlação positiva moderada quando considerada a amostra total do estudo $(p \leq 0,01)$.

Tabela 3 - Nível de correlação e significância entre as variáveis MET total, Índice de Massa Corporal, Coordenação global e Equilíbrio estático. Ivoti, RS.

\begin{tabular}{lcc}
\hline & \multicolumn{2}{c}{ MET total } \\
\cline { 2 - 3 } & Rho $(\varrho)$ & $P$ \\
\hline Faixa etária & $-0,219^{* *}$ & 0,002 \\
IMC & $-0,241^{* *}$ & 0,001 \\
Coordenação global & $0,274^{* *}$ & 0,000 \\
Equilíbrio estático & $0,191^{* *}$ & 0,006 \\
\hline
\end{tabular}

${ }^{* *} p \leq 0,01$

A tabela 4 apresenta os níveis de correlação entre a variável IMC, idade, coordenação global e equilíbrio estático dos indivíduos como um todo. Estes permitem observar que nos indivíduos suficientemente ativos houve correlação negativa entre as variáveis IMC e coordenação global $(p \leq 0,05)$. Nos indivíduos classificados como "insuficientemente ativos" e "muito ativos", ocorreu correlação negativa da variável IMC com coordenação global e IMC com equilíbrio. Em todas as classificações não foi identificada correlação entre a variável IMC e a faixa etária. 
Tabela 4 - Nível de correlação e significância entre a variável IMC, Coordenação global dos sujeitos divididos pelas classificações da variável MET (suficientemente ativos, insuficientemente ativos, muito ativos). Ivoti, RS.

\begin{tabular}{|c|c|c|c|c|c|c|}
\hline & \multicolumn{6}{|c|}{ MET } \\
\hline & \multirow{2}{*}{\multicolumn{2}{|c|}{$\begin{array}{c}\text { Suficientemente } \\
\text { Ativo }(\mathrm{n}=58) \\
\text { IMC }\end{array}$}} & \multirow{2}{*}{\multicolumn{2}{|c|}{$\begin{array}{c}\text { Insuficientemente } \\
\text { Ativo }(\mathrm{n}=65) \\
\text { IMC }\end{array}$}} & \multirow{2}{*}{\multicolumn{2}{|c|}{$\begin{array}{l}\text { Muito Ativo } \\
(\mathrm{n}=79) \\
\text { IMC }\end{array}$}} \\
\hline & & & & & & \\
\hline & Rho (@) & $P$ & Rho (@) & $P$ & Rho (@) & $P$ \\
\hline Idade & 0,090 & 0,500 & 0,172 & 0,169 & $-0,031$ & 0,784 \\
\hline Coordenação global & $-0,303^{*}$ & 0,021 & $-0,456^{* *}$ & 0,000 & $-0,416^{* *}$ & 0,000 \\
\hline Equilíbrio & $-0,215$ & 0,106 & $-0,390 * *$ & 0,001 & $-0,282^{*}$ & 0,012 \\
\hline
\end{tabular}

${ }^{*} p \leq 0,05 * * p \leq 0,01$

A tabela 5 demonstra os níveis de correlação entre a variável idade, IMC, coordenação global e equilíbrio estático dos indivíduos como um todo. Estes permitem observar que nos indivíduos suficientemente ativos houve correlação negativa entre as variáveis idade e equilíbrio estático $(p \leq 0,05)$. Nos indivíduos classificados como "insuficientemente ativos" e "muito ativos", ocorreu correlação negativa da variável idade com coordenação global e idade com equilíbrio.

Tabela 5 - Nível de correlação e significância entre a variável faixa etária, IMC, AM2 e AM3 dos sujeitos divididos pelas classificações da variável MET (AS, IA, MA). Ivoti, RS.

\begin{tabular}{|c|c|c|c|c|c|c|}
\hline & \multicolumn{6}{|c|}{ MET } \\
\hline & \multirow{2}{*}{\multicolumn{2}{|c|}{$\begin{array}{l}\text { Suficientemente } \\
\text { Ativo }(\mathrm{n}=58) \\
\text { Idade }\end{array}$}} & \multirow{2}{*}{\multicolumn{2}{|c|}{$\begin{array}{c}\text { Insuficientemente } \\
\text { Ativo ( } \mathrm{n}=65 \text { ) } \\
\text { Idade }\end{array}$}} & \multirow{2}{*}{\multicolumn{2}{|c|}{$\begin{array}{l}\text { Muito Ativo } \\
\quad(\mathrm{n}=79) \\
\text { Idade }\end{array}$}} \\
\hline & & & & & & \\
\hline & Rho (e) & $P$ & Rho (e) & $P$ & Rho (e) & $P$ \\
\hline IMC & 0,090 & 0,500 & 0,172 & 0,169 & $-0,031$ & 0,784 \\
\hline Coordenação global & $-0,256$ & 0,053 & $-0,317 *$ & 0,010 & $-0,285^{*}$ & 0,011 \\
\hline Equilíbrio & $-0,327 *$ & 0,012 & $-0,388^{* *}$ & 0,001 & $-0,247 *$ & 0,028 \\
\hline
\end{tabular}

${ }^{*} p \leq 0,05{ }^{* *} p \leq 0,01$

A tabela 6 apresenta os níveis de correlação entre as variáveis MET total com IMC, coordenação global e equilíbrio estático, considerando a divisão entre os gêneros e os indivíduos como um todo. É possível identificar que houve correlação negativa moderada entre as variáveis MET total e IMC, mas houve correlação positiva entre a variável MET total e coordenação global, assim como entre MET total e equilíbrio $(p \leq 0,01)$ na amostra geral. Quando analisado apenas o grupo masculino, nota-se que foi encontrada correlação negativa estatisticamente significativa entre as variáveis MET total e IMC. No grupo das mulheres participantes, maioria da amostra, é possível verificar o mesmo resultado da amostra total. 
Tabela 6 - Nível de correlação e significância entre as variáveis MET total, IMC, AM2 e MC e AM3 dos sujeitos divididos por sexo ( $\mathrm{n}=202)$. Ivoti, RS.

\begin{tabular}{lcccccc}
\hline & \multicolumn{2}{c}{ IMC } & \multicolumn{2}{c}{ Coordenação global } & \multicolumn{2}{c}{ Equilíbrio } \\
MET total & Rho $(\varrho)$ & $P$ & Rho $(\varrho)$ & $P$ & Rho $(\varrho)$ & $P$ \\
\cline { 2 - 7 } Homens $(\mathrm{n}=62)$ &,$- 294^{*}$ &, 020 &, 184 &, 152 &, 164 &, 202 \\
Mulheres $(\mathrm{n}=140)$ &,$- 212^{*}$ &, 012 &, $337 * *$ & 0,000 &, $206^{*}$ &, 015 \\
Total $(\mathrm{n}=202)$ & $-0,241^{* *}$ & 0,001 & $0,274^{* *}$ & 0,000 & $0,191^{* *}$ & 0,006 \\
\hline${ }^{*} \leq 0,05 * * p \leq 0,01$ & & & & & &
\end{tabular}

\section{DISCUSSÃO}

Comparando-se os resultados obtidos com dados do IBGE, ${ }^{13}$ no que se refere ao gênero, observa-se certa semelhança, já que destaca a prevalência da população idosa feminina $(69,3 \%)$ em relação à masculina (30,7\%). Camarano ${ }^{14}$ atribui essa diferença na composição por sexo ao fato de a população feminina possuir maior longevidade, sendo que quanto maior a idade da população de estudo, maior será a proporção de mulheres na mesma.

Considerando-se as áreas motoras específicas da escala, coordenação global e equilíbrio estático, para o teste de coordenação global, os participantes do estudo apresentaram média inferior quando comparada à média da variável equilíbrio, sendo que foram classificadas como "muito inferior" e "inferior", respectivamente, de acordo com os pontos de corte propostos pelo instrumento. Ambas as variáveis apresentam considerável variação em relação à média, onde a coordenação global apresenta variação (CV) superior a $50 \%$ da média (tabela 1).

Em estudo realizado com 142 idosos, de ambos os gêneros, e com idades entre 70 e 79 anos, ${ }^{15}$ também foram verificados padrões motores abaixo da normalidade nos testes de coordenação global e equilíbrio. Tais resultados foram também encontrados em 2007, quando Liposcki $^{16}$ estudou 50 idosos acima de 80 anos utilizando a mesma metodologia para a verificação da aptidão motora dos indivíduos.
A associação do aumento do IMC com riscos à saúde é contínua, mas de acordo com as populações analisadas, a interpretação dos dados difere, uma vez que os valores de referência são diferentes para determinadas faixas etárias, como também para os gêneros.

A coordenação global obteve menor média, 58,6 pontos, apresentando um desvio padrão de 30,56 e valores mínimo e máximo de 12 e 120, correspondendo a um nível "muito inferior" de aptidão nesta área motora, de acordo com o instrumento utilizado. Segundo Fonseca, ${ }^{17}$ a coordenação global é afetada durante o envelhecimento por fatores como a diminuição da tonicidade muscular, responsável pela manutenção das funções biológicas e psicológicas. Sendo assim, o baixo nível energético dificulta a manutenção da posição ortostática do idoso, sem que o mesmo acuse fadiga.

Quanto ao equilíbrio, atingiu-se uma média de 73,75 pontos, apresentando desvio padrão de 35,14 e valores mínimo e máximo de 12 e 132, classificando os participantes como "inferior". Segundo Ruwer, Rossi \& Simon, ${ }^{18}$ o desequilíbrio é um dos principais fatores que limitam a vida dos idosos, devido a um comprometimento do sistema de equilíbrio que ocorre com o envelhecimento. Entre 65 e 75 anos, cerca de 30\% dos idosos apresentam sintomas de desequilíbrio.

No processo de envelhecimento, é comum destacar-se, também, as doenças crônicas degenerativas que levam a alterações em vários 
órgãos do idoso, bem como distúrbios de postura e equilíbrio. ${ }^{19}$

Em relação ao IMC, nota-se que a média obtida pela amostra do estudo se encontra superior $(\bar{X}=28,9 \pm 4,89)$ ao considerado ideal para a população em questão. Segundo estudo de Lipschtz, ${ }^{12}$ no qual são sugeridas classificações específicas para a população idosa em relação ao estado nutricional, o ideal seria a manutenção do IMC entre 22 e $27 \mathrm{~kg} / \mathrm{m}^{2}$. A partir do momento em que o IMC passa de $27 \mathrm{~kg} / \mathrm{m}^{2}$, o indivíduo idoso é considerado obeso. Nos idosos, o ganho de peso pode aumentar devido ao declínio da função física que normalmente é observado com o aumento da idade, levando a uma limitação de movimentos e menor consumo energético. ${ }^{20}$ Spirduso, Francis \& MacRae ${ }^{21}$ afirmam que, assim como mudanças na dieta e no nível de atividade física, o padrão genético e a interação entre esses fatores também parece influenciar no ganho de peso e no acúmulo de gordura corporal que surge ao longo da idade.

É possível observar que houve correlação negativa moderada entre as variáveis IMC e coordenação global, assim como entre IMC e equilíbrio, quando considerada a amostra total do estudo. Quando estratificados por sexo, essas associações não foram significativas nos homens; entretanto, entre as mulheres da amostra, o alto valor do IMC pode ter gerado resultados inferiores no desempenho dos testes de coordenação global e equilíbrio (tabela 2).

Rebelatto et al. ${ }^{22}$ estudaram 303 mulheres $(62,97 \pm 7,59$ anos) e 51 homens $(65,69 \pm 7,49$ anos), a fim de analisar o equilíbrio estático e o dinâmico, e verificar sua associação com a idade e com o IMC. De acordo com os resultados obtidos, os autores concluíram que no grupo feminino, o IMC elevado esteve associado a déficits de equilíbrio estático e dinâmico, enquanto que no grupo masculino, não foi observada a mesma correlação. Em relação ao sexo feminino, resultados semelhantes foram observados por Apovian et al., ${ }^{23}$ que observaram que o valor elevado de IMC se associou a um pior desempenho no Teste de Apoio Unipodal, quando estudaram 90 idosas com idade média de $71 \pm 4.9$ anos.

Em estudo de Mazo et al., avaliaram-se 52 indivíduos do sexo feminino, com idade média de 68,62 \pm 4,98 anos, com o objetivo de verificar a relação entre o IMC e o Índice de Aptidão Funcional Geral (IAFG). A partir da análise dos resultados, os autores observaram uma tendência de $42,9 \%$ das idosas que apresentaram IAFG bom apresentarem um IMC normal, e de 95,6\% das idosas com IAFG fraco de se apresentarem classificadas como sobrepeso em relação ao IMC. Por outro lado, Era et al. ${ }^{24}$ observaram associação entre alto IMC e bom desempenho nos testes de equilíbrio estático e dinâmico em idosas com 75 anos de idade.

Napresenteinvestigação,pode-seobservarque a idade não está relacionada ao IMC nos diferentes níveis de atividade física. A coordenação global está relacionada negativamente ao IMC em todos os níveis de atividade física. Mas é interessante observar que o equilíbrio está correlacionado negativamente ao IMC nas pessoas muito ativas ou insuficientes ativas, mas não nas pessoas suficientemente ativas. De forma geral, segundo Spirduso, ${ }^{3}$ o envelhecimento acarreta perda da estabilidade das funções, causando dificuldade para responder a diferentes estímulos e limitando progressivamente a capacidade da pessoa idosa de empreender as atividades de vida diária. Da mesma forma, a obesidade está relacionada com limitações funcionais, diminuição da qualidade de vida e fatores de instabilidade. ${ }^{23}$

A partir de todos os dados levantados, é importante considerar as diferenças entre $\mathrm{O}$ sexo masculino e feminino que se apresentam nos objetivos propostos pelo presente estudo. Os homens tendem a aumentar o IMC em idades inferiores ao estudo em questão, como apontam os estudos de Spirdus, ${ }^{3}$ ao contrário das mulheres, que tendem ao aumento da incidência da obesidade na faixa etária alvo do estudo. $\mathrm{Na}$ faixa etária dos 60 anos aos 69 anos, as pessoas apresentam um desconforto por estarem entrando na faixa do ciclo vital relacionada à velhice. Assim, 
muitos comportamentos e sintomas psicológicos como depressão demonstram elevação, com uma sequência de declínio nas faixas etárias subsequentes. Pode-se compreender este momento como uma acomodação ao novo status da identidade de idoso. ${ }^{25}$

As mulheres sofrem com o sobrepeso e o desajuste corporal, assim como com o desconforto emocional e social decorrente da mudança da autoimagem que as caracterizava até o momento. As alterações de identidade visual são mais complexas para as mulheres do que para os homens no decorrer do processo de envelhecimento. Esta situação muitas vezes leva a problemas emocionais, como baixa na autoestima e sintomas de depressão situacional. ${ }^{26}$

Seria possível relacionar as dificuldades apresentadas pelas idosas na coordenação global e no equilíbrio estático ao sedentarismo identificado no presente estudo. A coordenação global e o equilíbrio estão relacionados positivamente ao nível de atividade física apenas nas mulheres. Entretanto, o IMC está relacionado negativamente ao nível de atividade física em ambos os gêneros. Possivelmente, as questões de mudanças físicas e sua consequente relação na autoimagem sejam mais significativas nas mulheres, podendo agravar vários elementos da qualidade de suas vidas.

Os dados da OMS $^{9}$ apontam a relevância de se compreender as relações existentes entre um envelhecimento ativo e questões como obesidade, possibilidade de realizar atividades físicas e capacidade funcional. Neste sentido, pode-se entender que ao se realizar um controle da obesidade feminina na velhice, se estará prevenindo e melhorando sua saúde mental e frequentemente promovendo contatos sociais.

Neste processo, acaba ocorrendo um ciclo vicioso de envelhecimento, pois as mulheres tendem a realizar menos atividades físicas para não exporem seu corpo e, assim, diminuírem a possiblidade de desenvolver as habilidades de equilíbrio e de coordenação global que seriam de grande importância nessa fase de suas vidas. Esta prevenção auxiliaria as mulheres idosas a ficarem mais independentes o máximo de tempo possível, uma vez que elas costumam viver mais do que seus cônjuges. Identificou-se nas estatísticas ${ }^{25}$ tendência de os idosos residirem sozinhos, o que, diante dos dados apresentados, demonstra que as mulheres terão mais dificuldades de continuarem residindo sozinhas do que os homens, em função do risco de quedas ou de acidentes.

A idade não demonstrou correlação significativa com o IMC quando a amostra foi dividida de acordo com o nível de atividade física; por outro lado, a variável idade demonstrou correlação negativa com o equilíbrio em todos os níveis de atividade física. Entretanto, a variável idade apresentou correlação negativa com a coordenação global nos grupos muito ativos e insuficientemente ativos, mas não no grupo suficientemente ativo. Os dados demonstram a necessidade, como aponta a OMS, ${ }^{9}$ de estudos relacionados à longevidade.

Portanto, segundo a OMS, ${ }^{9}$ há importantes benefícios econômicos com o gasto na área da saúde e também na área social quando os idosos são fisicamente ativos. A queda de pessoas idosas é causa crescente de lesões, custos de tratamento e principalmente preocupante de óbitos. As lesões sofridas por idosos são de maior gravidade, pois levam à situação de dependência física, social e emocional, além de períodos de internação e reabilitação prolongados.

Os dados do presente estudo corroboram estudos anteriores e servem de base para a continuidade de novos focos de investigação, uma vez que se percebe que, além das dificuldades que podem ser impostas pelo avanço da idade, existem ainda percepções pessoais do envelhecimento corroboradas pela percepção social. Ou seja, muitas das fragilidades podem ser prevenidas com mudanças de atitudes. Entretanto, como aponta a OMS, ${ }^{9}$ a percepção tradicional de que as fragilidades do idoso e as quedas sejam "acidentes, resultou em uma negligência histórica nessa área da saúde pública” (p.28). 


\section{CONCLUSÃO}

A partir deste estudo, conclui-se que houve correlação negativa significativa moderada entre o IMC e a coordenação global e o equilíbrio para os indivíduos idosos do sexo feminino. Assim, quanto maior for o resultado da variável IMC, menores serão os resultados dos testes relacionados à coordenação global e ao equilíbrio estático nos indivíduos do sexo feminino participantes do estudo. Uma correlação negativa significativa moderada foi também constatada entre o nível de atividade física em METs totais e IMC entre homens e mulheres. Entretanto, constatou-se que os resultados referentes ao

\section{REFERÊNCIAS}

1. Matsudo SM, Matsudo VK, Matsudo R, Barros Neto TL. Impacto do envelhecimento nas variáveis antropométricas, neuromotoras e metabólicas da aptidão física. Revista brasileira de ciência \& movimento. 2000 set; 8(4):21-32.

2. Okuma SS. O idoso e a atividade física: fundamentos e pesquisa. Campinas: Papirus; 1998.

3. Spirduso WW. Dimensões físicas do envelhecimento. Trad. Paula Bernardi. Barueri (SP): Manole; 2005.

4. Rosa Neto F, et al. Manual de avaliação motora para a terceira idade. Porto Alegre: Artmed; 2009.

5. World Health Organization. Global strategy on diet, physical activity and health. Disponível em: <http:// www.who.int/dietphysicalactivity/publications/facts/ obesity/en. 2010>.

6. World Health Organization. Obesity and overweight. Fact Sheet WHOI311. set/2006. Disponível em: <http://whqlibdoc.who.int/fact_ sheet/2006/FS_311.pdf $>$.

7. Mazo GZ, Kulkamp W, Lyra VB, Prado AP. M. Aptidão funcional geral e o índice de massa corporal de idosas praticantes de atividade física. Revista brasileira de cineantropometria \& desempenho humano. 2006; 8(4):46-51.

8. Cupertino APFB, Rosa FHM, Ribeiro PCC. Definição de envelhecimento saudável na perspectiva de indivíduos idosos. Psicologia, Reflexão e Critica. [online]. 2007; 20(1):816. Disponível em: <http://www.scielo.br/ scielo.php?script $=$ sci_arttext\&pid $=\mathrm{S} 0102$ 79722007000100011\&lang=pt\&tlng=pt $>$. nível de atividade física em METs totais estavam correlacionados positivamente com os resultados dos testes de coordenação global e equilíbrio na amostra total do estudo.

Pelo fato de o presente estudo tratar de uma pesquisa descritiva, de corte transversal, que pressupõe a coleta de dados num único momento, sem a possibilidade de um seguimento na investigação, sugere-se que sejam conduzidos novos estudos que utilizem outros delineamentos de pesquisa que prevejam intervenções e grupo controle. Neste caso, poder-se-ia analisar mais profundamente as variáveis do estudo buscandose relações de causa e efeito entre elas.

9. World Health Organization. Envelhecimento ativo: uma política de saúde. Brasília: Organização PanAmericana da Saúde (OPAS); 2005.

10. Centro de Estudos do Laboratório de Aptidão Física de São Caetano do Sul. Questionário Internacional de Atividade Física - versão curta. Disponível em: http://www.celafiscs.institucional.ws/65/ questionarios.html. Acesso em: 24 abr. 2011.

11. International Physical Activity Questionnaire Group. Guidelines for Data Processing and Analysis of the International Physical Activity Questionnaire (IPAQ) - Short and Long Forms November 2005. Available at: http://www.ipaq.ki.se/scoring.pdf.)

12. Lipschitz DA. Screening for nutritional status in the elderly. Primary Care. 1994; 21(1):55-67.

13. IBGE. Projeção da população do Brasil, Comunicação Social, 27 de novembro de 2008. Disponível em: <http://www.ibge.gov.br/home/ presidencia/noticias/noticia_visualiza.php?id_ noticia $=1272 \&$ id_pagina $=$ e1 $>$.

14. Camarano AA. Envelhecimento da população brasileira: uma contribuição demográfica. Instituto de Pesquisa Econômica Aplicada, Rio de Janeiro; 2002. Disponível em: <http://www.ipea.gov.br/pub/ td/td_2002/td_0858.pdf $>$.

15. Rosa Neto F, Liposcki DB, Teixeira CAA. Estudo dos parâmetros motores em idosos com idade entre 70 e 79 anos pertencentes aos grupos da terceira idade da prefeitura de São José - SC. Efdeportes Revista Digital 2006; 10(92):1. Disponível em: $<$ http://www.efdeportes.com/efd92/idosos.htm>. 
16. Liposcki DB. A influência de um programa de intervenção psicomotora na aptidão motora de idosos longevos. [dissertação]. Florianópolis: Universidade do Estado de Santa Catarina, Curso de Mestrado em Ciências do Movimento Humano; 2007.

17. Fonseca V. Manual de observação psicomotora: significação psiconeurológica dos fatores psicomotores. Porto Alegre (RS): Artes Médicas; 1995.

18. Ruwer SL, Rossi AG, Simon LF. Equilíbrio no idoso. Rev Bras Otorrinolaringol. 2005; 71(3):298-303.

19. Gonçalves DFF, Ricci NA, Coimbra AMV. Equilíbrio funcional de idosos da comunidade: comparação em relação ao histórico de quedas. Rev Bras Fisioter. 2009 jul./ago.; 13(4):316-23.

20. Mello VDF. Obesidade. In: Busnello FM. Aspectos Nutricionais no Processo do Envelhecimento. São Paulo: Atheneu; 2007.

21. Spirduso WW, Francis KL, Macrae PG. Physical Dimensions of Aging. 2. ed. Champaign: Human Kinetics; 2005.
22. Rebelatto JR, Castro AP, Sako FK, Aurichio TR. Equilíbrio estático e dinâmico em indivíduos senescentes e o índice de massa corporal. Fisioterapia em movimento. 2008; 21(3):69-75.

23. Apovian CM, Frey CM, Wood GC, Rogers JZ, STILL CD, Jensen GL. Body mass index and physical function in older women. Obes Res. 2002 Aug;10(8):740-7.

24. Era P, Schroll M, Ytting H, Gause-Nilsson I, Heikkinem E, Steen B. Postural balance and its sensory-motor correlates in 75-year-old men and women: a cross-national comparative study. J Gerontol A Biol Sci Med Sci.1996 Mar;51(2):M53-63.

25. Santos GA, VAZ CE. Planejamento, ansiedade e tensões psíquicas na terceira idade, avaliadas através do Rorschach. Psico. 1997; 28(1):187-206.

26. Santos GA. Os conceitos de saúde e doença na representação social da velhice. Textos \& Contextos. 2002; 1(1):1-12.

27. IBGE. Perfil dos idosos responsáveis pelos domicílios no Brasil. Rio de Janeiro; 2002. 